\title{
Primary Healthcare Payer at Diagnosis
}

National Cancer Institute

\section{Source}

National Cancer Institute. Primary Healthcare Payer at Diagnosis. NCI Thesaurus. Code C160898.

Primary payer/insurance carrier at the time of initial diagnosis and/or treatment. 